\title{
Doing It Right: Combining Edutainment Format Development and Research
}

\author{
Simon Staffans ${ }^{1}$, Annika Wiklund-Engblom ${ }^{1}$, Marc Hassenzahl ${ }^{1,2}$, \\ and Susanne Sperring ${ }^{1}$ \\ ${ }^{1}$ MediaCity, Åbo Akademi University, Box 311, 65101 Vaasa, Finland \\ ${ }^{2}$ Folkwang University, Campus Universitat Duisburg-Essen, Universitatstrasse 12, 45141 \\ Essen, Germany \\ \{Simon.Staffans, Annika.Wiklund-Engblom, Susanne.Sperring\} @abo.fi, \\ Marc.Hassenzahl@folkwang-hochshule.de
}

In this paper, we elaborate on the synergy achieved from combining cross media content development and research. We describe the cross media edutainment format "The Space Trainees" (TST), its development, and how we involve research as a tool for assisting the design process. This is the first and foremost aim of the research attempts, in which targets are both usability of the format and user experience (UX). Another aim of involving research is to gain more understanding of the edutainment value this cross media format delivers; i.e. both the value of entertainment and learning. The third aim is to fine-tune research instruments to better target children's UX, with regard to their attitudes, emotions, actions, and reactions.

The Space Trainees has been developed utilizing the principles of bridged cross media, i.e. the development of a storyline that continues in a logical way from one media - the television show - to another- the web portal - and back again. The development work has throughout the process of the project been aimed at producing a viable commercial cross media edutainment format for production and international marketing. The research involved in the development work is conducted in convergence with the design phases, and hence, the aims are always pragmatic (see figure). One crucial factor has been the correct timing of the tests.

The final cross media product is released in the fall of 2009. The final product will be subjected to research targeting its value for learning and entertainment (study

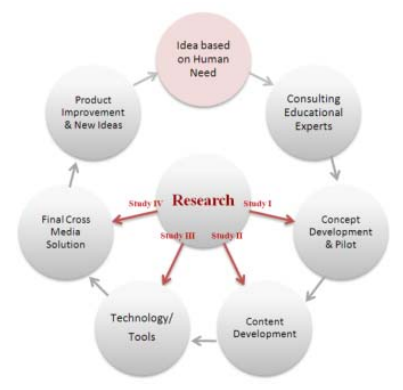
IV). The aim of this research is to build know-how for the cross media design team for future edutainment projects, but also to investigate further into the educational perspective on combining learning and play.

During the format development phases of TST research into both usability issues and UX has supported the development work. The first study gave indications that the interactivity was well received by viewers and users. The overall concept also received support. Interactivity via set-top-box, however, brought some negative issues that contributed to the switch to cross media solutions instead. Study two gave much needed information on the set instructions, the games themselves and the collaboration between the children on the television show, and contributed to changes made during the later development phases. 